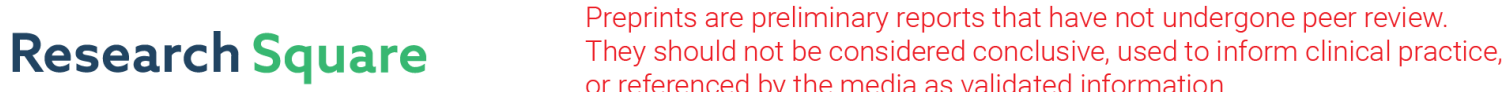 or referenced by the media as validated information. \\ The Sequence of the Ribosomal Binding Site Controls the Gene Expression in Brucella
}

\section{Shuang Liu}

Jilin Agricultural University

Peng Wang

Beijing Institute of Biiotechnology

\section{Yanbo Dong}

Capital Medical University Affiliated Beijing Friendship Hospital

\section{Heling Xu}

Shenyang Agricultural University

\section{Shanhu Li}

Beijing Institute of Biotechnology

Linbo Zhang ( $\nabla$ cczlb@126.com )

Jilin Agricultural University https://orcid.org/0000-0003-3627-6757

\section{Research}

Keywords: Brucella, Ribosome binding sites, Shine Dalgarno, spacer, Lipopolysaccharide

Posted Date: October 13th, 2021

DOl: https://doi.org/10.21203/rs.3.rs-944639/v1

License: (c) (i) This work is licensed under a Creative Commons Attribution 4.0 International License. Read Full License 


\section{Abstract}

\section{Background}

Brucella is an important pathogen causing Brucellosis. Vaccine strains obtained by a single knockout cannot combine low virulence and immunogenicity. Our study modified the SD sequence and spacer sequence of the RBS of Brucella to affect its protein expression. We altered the RBS of LPS-associated genes to reduce LPS-associated protein expression while retaining LPS integrity.

Results

We first established an evaluation system based on the reporter gene red fluorescent protein mCherry. The mCherry expression could be changed by altering the Shine Dalgarno sequence and spacer sequence of RBS. After optimizing the Shine Dalgarno sequence, mCherry expression was increased 4-fold in $E$. coli and decreased by $1 / 4$ in Brucella. The mCherry expression was increased 1.5-fold in E. coli and decreased to $1 / 2$ in Brucella when the length of the spacer sequence was 0 . When the spacer sequence was NA ( $\mathrm{N}=$ $4,8,12 \mathrm{nt})$ or NG (N =4, 8, 12nt), mCherry expression was reduced in both E. coli and Brucella. Accordingly, two mutant strains were constructed in an attempt to decrease the expression of $L p t A$ and LpxO, Brucella LPS-related genes, by $1 / 4$. Silver staining experiments of LPS SDS-PAGE revealed an alteration in the composition of LPS in the two mutant strains. Polymyxin B experiments revealed that both mutant strains were more sensitive to Polymyxin B resistance.

Conclusion: In Brucella, the expression of the target gene could be affected by changing the length or the composition of the RBS sequence. The LPS gene remained unchanged while reducing the expression of its associated protein, achieving the original goal of reducing bacterial virulence while retaining immunogenicity. It is a promising strategy to improve the safety and efficacy of vaccines.

\section{Background}

Brucella is a Gram-negative bacterium that causes brucellosis, a widespread zoonotic disease [1-5]. By expressing different virulence factors and using various strategies to escape the host's immune response, Brucella is able to survive and replicate in host cells without being completely wiped out by the host, allowing Brucellosis to progress from an acute stage to chronic infection [6].

Lipopolysaccharide (LPS), a major component of the cell wall of Gram-negative bacteria, is an important virulence factor for Brucella [7]. LPS is involved in bacterial survival in many hosts, contributing to complement and bactericidal peptide resistance and to bacterial adherence and entry into cells [8]. LPS contains a marked pathogen associated molecular pattern (PAMP) that can be recognized by immune cells and body fluids. It also triggers specific antibody responses and protective immunity against pathogens [9]. At the same time, LPS provides a permeable barrier that makes an important contribution to the structural integrity of the bacteria, which is linked to their viability. 
LPS is constituted by a hydrophobic lipid A linked to a short, usually negatively charged, oligosaccharide core followed (smooth LPS) or not (rough LPS) by a hydrophobic 0-polysaccharide (0-chain or 0antigen) [7]. However, some species of Brucella are of weak virulence. Bacterial virulence can be altered by deleting certain genes involved in the LPS modification regulatory pathway $[7,10]$. However, this knockdown approach also reduces the immunogenicity of the strain, thus weakening the protective effect of the vaccine. Therefore, reducing virulence and retaining some immunogenicity by decreasing the protein expression of LPS-related genes would be an effective vaccine design attempt.

Gene expression requires efficient translation initiation. The initiation process is characterized by the formation of the $30 \mathrm{~S}$ initiation complex, including the $30 \mathrm{~S}$ ribosomal subunit, three initiation factors, the fMet-tRNA, and the mRNA at the ribosomal binding site (RBS) [11]. In bacteria, proteins are synthesized by ribosomes according to instructions encoded by mRNA sequences [12-15]. RBS affects the translation process [16]. The non-coding regions of mRNA, especially the 5'-untranslated region (5'-UTR), contribute significantly to the efficiency of protein synthesis $[17,18]$. The most important 5 -UTR sequence elements in bacterial mRNAs were Shine-Dalgarno (SD) sequences, the 16S rRNA binding sites, and the spacer between the target gene start codon and the SD sequence $[19,20]$. The same RBS sequence in different genetic backgrounds can also lead to large differences in protein expression levels.

The folding of transcripts into secondary structures in the RBS region is critical for the initiation of translation. Stable structure in this region may prevent ribosomes from entering the transcripts and reduce the rate of translation initiation $[21,22]$. The spacer between the start codon and the SD sequence is a component of the RBS and is therefore expected to affect the folding of transcripts in this region [2327]. The optimum distance between the SD cassette and the start codon was 5-9 nt in E. coli $[28,29]$ and 7-8 nt in B. subtilis [30]. It is demonstrated that the composition of the spacer sequence has a significant effect on the expression yield. The expression level is determined not only by the length but also by the different nucleotide composition of the spacer bars [30].

Bacterial protein synthesis systems are one of the main targets of biotechnological modification. The functional evaluation of protein 5'-UTR sequence elements is usually performed by monitoring the expression of their downstream reporter gene[31]. In this study, the mCherry reporter gene was introduced into Brucella genome in order to analyze how much the spacer sequence of RBS affects gene expression in Brucella, based on our experience of $E$. coli. It was confirmed that the number of bases and base composition of the RBS spacer in Brucella affects the expression of the downstream reporter gene mCherry. The patterns were applied to the RBS of Brucella LPS-related genes. They caused a reduction in the expression level of the Brucella LPS-related genes and thus hampered the integrity of LPS.

\section{Results And Discussion}

\section{Construction of standard expression plasmids}

SecE is a non-essential protein translocase from Brucella abortus 2308 vaccine strain 104M. The expression of $\operatorname{Sec} E$ is controlled by a strong Brucella spp. promoter called PsojA[32, 33]. The RBS 
sequence of SecE gene is GAGGTTGTGCCTGATCAGA constituted by the SD sequence GAGGTTGTGCCTGAT and the spacer sequence CAGA. The mCherry protein is a red fluorescent protein[34]. Accordingly, an RBS alteration evaluation system based on mCherry red fluorescent protein was established to determine the effect of RBS alteration on Brucella gene expression. Sequentially ligate the mCherry gene, the homologous arm of SecE and Kan resistance by Overlap PCR. Clone the ligation onto the pBM16A T vector and the acquired new plasmid was named as $\mathrm{pMCH}$. The 5'-flanking region of $S e c E$ gene contained PsojA and RBS sequence of $\operatorname{SecE}$ gene. The $\mathrm{pMCH}$ is a shuttle plasmid. It not only contains the origin of E. coli, but also is able to recombine in Brucella. Transform pMCH into E. coli, and the new strain was named E. MCH. Integrate $\mathrm{pMCH}$ into the genome of Brucella 104M to replace the SecE gene, and the new strain was named B. MCH (Fig. 1a). The SD sequence of mCherry is GAGGTTGTGCCTGAT and the spacer sequence is CAGA in E. MCH and $\mathrm{B}$. $\mathrm{MCH}$. Due to the expression of mCherry protein, $\mathrm{E} . \mathrm{MCH}$ and $\mathrm{B}$. $\mathrm{MCH}$ were observed to be red by naked-eye, fluorescence microscope and multi-function imager. Red arrow indicates one colony. (Fig. 2). B. $\mathrm{MCH}$ and E. MCH transformants emitted bright red fluorescence under laser confocal microscopy. These fluorescence were distributed throughout the cells as small red dots, whose position and size were consistent with the strain. It is noteworthy that the red fluorescence of the wild strain of $E$. coli is brighter than that of Brucella. This may be due to the fact that mCherry is present in E. coli as a multi-copy plasmid, while in Brucella it is present as a single copy integrated into the genome.

\section{SD sequences influence the expression of mCherry}

Replace the SD sequence GAGGTTGTGCCTGAT of mCherry with AAAGGAGGT, and the new plasmid was named as $\mathrm{pMCH} 1$. Transform pMCH1 into $E$. coli, and the new strain was named $\mathrm{E}$. $\mathrm{MCH} 1$. Integrate pMCH1 into the genome of Brucella 104M to replace the SecE gene, and the new strain was named B. MCH1 (Fig. 1b).

We first observed qualitatively the intensity of fluorescence by confocal microscopy. The red fluorescence intensity of E. MCH1 was increased compared to E. MCH (Fig. 3a). On the other hand, B. MCH1 caused a decrease in the fluorescence intensity compared to $\mathrm{B}$. $\mathrm{MCH}$ (Fig. 3b). Then the fluorescence intensity of mCherry was quantitatively analyzed by a multi-functional microplate reader. The fluorescence intensity of $\mathrm{E} . \mathrm{MCH} 1$ increased to $432.235 \%$ of $\mathrm{E}$. MCH's and B. MCH1 decreased to $74.964 \%$ of B. MCH's, respectively (Fig. 3c, 3d). This may be due to the different nature in translation levels between $E$. coli and Brucella. The differences of red fluorescence intensity indicated the differential expression of mCherry protein. Thus, the changes of SD sequence influenced the expression of mCherry.

\section{Verification of the effect of RBS with different spacer length on gene expression}

It was shown that the expression level is determined not only by the length of the spacer sequence, but also by the different nucleotide composition of the spacer sequence [35]. The preliminary results of this experiment showed that the expression level of mCherry gene was not upregulated in Brucella after changing the optimal SD sequence. To determine the effect of spacer sequence of gene expression in 
Brucella, subsequent experiments were designed to modify the spacer sequence of RBS. The fluorescence intensities of $\mathrm{B}$. $\mathrm{MCH}$ and $\mathrm{E}$. $\mathrm{MCH}$ were used as negative controls.

We constructed and screened mCherry RBS for different spacer sequences $N A(N=0,4,8,12 n t)$ or NG (N $=0,4,8,12 \mathrm{nt})$. For this purpose, plasmids containing different spacer sequences were introduced into $E$. coli and Brucella. The new E. coli strains were named as E. MCHO, E. MCH4A, E. MCH4G, E. MCH8A, E. $\mathrm{MCH} 8 \mathrm{G}, \mathrm{E} . \mathrm{MCH} 12 \mathrm{~A}$, and E. MCH12G. The recombination Brucella strains were named as $\mathrm{B}$. MCH0, $\mathrm{B}$. MCH4A, B. MCH4G, B. MCH8A, B. MCH8G, B. MCH12A, and B. MCH12G (Fig. 1b). Their red fluorescence intensities were observed qualitatively and quantitatively.

The differences in fluorescence intensity were first observed by confocal microscopy. E. MCH4G caused a decrease in the fluorescence intensity and $\mathrm{E}$. $\mathrm{MCHO}$ was increased compared to $\mathrm{E}$. $\mathrm{MCH}$. Meanwhile, $\mathrm{B}$. $\mathrm{MCHO}$ caused a decrease in the fluorescence intensity compared to $\mathrm{B}$. $\mathrm{MCH}$. The fluorescence of other strains was not obvious (Fig. 4a, 4b).

Then the fluorescence intensities of mCherry were analyzed by a multi-functional microplate reader. The fluorescence intensity of $\mathrm{E}$. MCHO increased to $156.693 \%$ of E. MCH's. But E. MCH4G, E. MCH8A, and E. $\mathrm{MCH} 12 \mathrm{~A}$ showed decreased to $82.572 \%, 7.772 \%$, and $5.879 \%$ of $\mathrm{E}$. MCH's, respectively. And the fluorescence intensities of the other strains were reduced to 0 (Fig. 4c). What's more, the fluorescence intensity of $\mathrm{B}$. MCHO and B. MCH4G decreased to $52.532 \%$ and $25.135 \%$ of $\mathrm{B}$. MCH's. The fluorescence intensities of the other strains were reduced to 0 (Fig. 4d).

The results of fluorescence intensity observed by laser confocal microscopy and microplate reader were consistent. These experiments demonstrated that the composition and length of RBS spacer sequence had an influence on gene expression in Brucella.

\section{RBS influence the expression of Brucella LPS}

After obtaining the relationship between RBS and gene expression in Brucella abortus strain $104 \mathrm{M}$, to further explore the role of RBS alterations in different Brucella species, the experiment was subsequently validated in a Brucella Melitensis 16M wild strain. Previous studies on Brucella related genes were performed by knockout mutations of a single gene. The strategy reduced the virulence of Brucella, but also reduced the immunoprotective effect as well. In this study, the genetic integrity of the strain was preserved, and in order to retain some immunoprotective effects while reducing some virulence, the RBS designing which retains 3/4 of protein expression, was chosen to modify Brucella LPS-related genes.

$L p t A$ and $L p x O$, were closely related to the synthesis of LPS lipid A. It was shown that when two Brucella LPS genes, $L p t A$ and $L p x O$, were knocked out separately, the mutant strain was less virulent and more sensitive to Polymyxin B as LPS integrity reduced [10]. Therefore, $L p t A$ and $L p x O$ were chosen for this study, and the obtained mutant strains could be tested by Polymyxin B. 
The gene $L p t A$ and $\angle p x O$ were changed by an overlap extension PCR in the same way as described for $\mathrm{pMCH}$. The RBS of the $L p t A$ gene was altered and inserted $\mathrm{kan}^{\mathrm{r}}$ and the plasmid was named pA1 (Fig. 1c). The RBS of the $\angle p x O$ gene was altered and inserted $\mathrm{Gm}^{r}$ and the plasmid was named p01 (Fig. 1d). Since LPS is the product of multiple genes, we designed single-gene mutations and double-gene combination mutations in order to study the effect of RBS alteration of genes on LPS. After the transformation of Brucella 16M, two clones were screened on resistance plates, named B. A1 and B. A101.

To better investigate the relationship between the LPS content of Brucella and the RBS of the gene. The LPS of the mutant strains were extracted to conduct SDS-PAGE and gel silver staining. LPS consists of Oantigen, Core, and Lipid A. The breadth and color shades of the LPS bands were used as a measure of the integrity of the LPS. The 0-chain was related to the type of LPS of the strain, with more pronounced bands in the smooth strain and almost blank bands in the rough strain. The difference between the LPS bands of the mutant strains and the wild strain was observed (Fig. 5). The breadth and color of O-antigen, Core and Lipid A of the LPS of B. A1 and B. A101 were weaker than $16 \mathrm{M}$. This result corroborated the RBS sequence had an impact on the integrity of Brucella LPS.

When it comes to the function of LPS, an intact LPS gene contributed to Polymyxin $B$ resistance in Brucella. The killing efficacies of Polymyxin B on the mutant strain were examined by survival at concentrations of $50,100,200$, and $500 \mu \mathrm{g} / \mathrm{mL}$ for 1 to $5 \mathrm{~h}$. The results showed that the survival of Brucella gradually decreased as the increase of drug concentration and treatment time. The survival of mutant strains was decreased compared with that of Brucella wild strain 16M (Fig. 6). The mutant strains were more sensitive to Polymyxin B than wild strain B. melitensis $16 \mathrm{M}$.

In summary, the results of silver staining and Polymyxin B sensitivity test showed that B. A1 and B. A101 strains had weaker LPS integrity and drug resistance than wild strains. Preliminarily, the B. A1 and B. A101 mutant strains might be weak virulent strains of Brucella 16M strain. The virulence and immunogenicity of these three mutant strains will be further demonstrated by animal immunization experiments in future studies. This will further illustrate the relationship between RBS and bacterial virulence and immunogenicity.

\section{Conclusions}

Genetic modification is essential for Brucella studies. Modifying RBS is an effective way to alter gene expression. Our study evaluated the spacer sequence between SD sequence and the start codon to alter Brucella gene expression [21]. In this study, mCherry red fluorescent protein was used to exhibit visually the effect of RBS on Brucella gene expression. Accordingly, an RBS alteration evaluation system based on mCherry red fluorescent protein was established to determine the effect of RBS alteration on Brucella gene expression. Brucella strains with altered LPS gene expression was obtained after RBS transformation of Brucella LPS-related genes. In Brucella, the expression of the target gene could be affected by changing the length or the composition of the RBS sequence. The LPS gene remained 
unchanged while reducing the expression of its associated protein, achieving the original goal of reducing bacterial virulence while retaining immunogenicity. It is a promising strategy to improve the safety and efficacy of vaccines.

\section{Materials And Methods}

\section{Strains and plasmids}

E. coli strains were routinely cultured in Luria Bertani (LB) broth or agar at $37^{\circ} \mathrm{C}$. B. abortus vaccine strain $104 \mathrm{M}$ and $B$. melitensis strain $16 \mathrm{M}$ were cultured in trypticase soy agar (TSA) or trypticase soy broth (TSB) (BD, USA) at $37^{\circ} \mathrm{C}$ for 2-3 days. When mutation screening was required, $\mathrm{Kan}^{\mathrm{r}}$ or $\mathrm{Gm}^{\mathrm{r}}$ was added to the culture medium at a final concentration of $50 \mu \mathrm{g} / \mathrm{ml}$. Other strains were obtained as shown in Table 1 . Experimental operations related to Brucella $104 \mathrm{M}$ and $16 \mathrm{M}$ were performed in a Class $\otimes$ Biological Safety Cabinet (BSC). The plasmids used in this paper were shown in Table 1.

\section{Prediction of RBS sequence using Bioinformatics analysis}

The Ribosome Binding Site (RBS) Calculator can predict RBS sequences of a protein coding sequence in bacteria to rationally control the translation initiation rate. This tool was used to analysis the RBS sequence of Brucella. It is available at https://www.denovodna.com/software/ [36].

\section{Construction of standard expression plasmids}

To generate the standard expression plasmids, approximately $1 \mathrm{kbp}$ of 5 -flanking region and 3 '-flanking region of the SecE gene were amplified by PCR using the Brucella 104M genomic DNA as the template. The Kanamycin resistance gene (Kan') cassette was PCR amplified from pMAZ-SK plasmid[37]. The red fluorescence protein (mCherry) cassette was PCR amplified from pTREX-mCherry plasmid [38, 39]. PCR was performed using PrimeSTAR high fidelity DNA polymerase (Takara, Japan). The PCR fragments were fused by overlap extension PCR and cloned into the pBM16A T vector冈Biomed, China冈for sequencing. The recombinant vectors were designated $\mathrm{pMCH}$. The plasmids were verified by PCR and automated sequencing analysis. The primers are shown in Table S1. The plasmid pMCH1, pA1, and pA101 was constructed in the same way (primers are shown in Table S1).

\section{Construction of different spacer sequence lengths of plasmids}

The amplified fragment includes an RBS sequence that was amplified as Aflll / Pstl fragments using the primers SP1 and SP2 (Table S1) from the pMCH1 plasmid. The spacer sequence CAGA of Aflll / Pstl fragments was replaced by $N A(N=0,4,8,12 n t)$ or $N G(N=0,4,8,12 n t))$ using Aflll and Mscl restriction enzymes with the oligos Sp3-Sp16 (Table S1) to obtain fragments with different spacer sequences. Subsequently, the pMCH1 plasmid was hydrolyzed using the restriction enzymes Afllll and Pstl, and fragments with different spacer sequences were ligated into the $\mathrm{pMCH} 1$ plasmid. The acquired plasmids series contains different spacer sequences. 


\section{Transformation of E. coli and Brucella}

The target fragments were cloned into a pBM16A T vector (Biomed, China). The plasmids were transformed into chemical competent DH5a. Pick up the single colony for sequencing (Biomed, China) (The E. colistrain obtained is shown in Table 1 for comparison with Brucella). The plasmid DNA was isolated and transformed into Brucella 104M or 16M electro-transformed competent, and was screened for resistance. By using the homologous recombination function of Brucella itself, the homologous parts of the plasmid were integrated into the chromosome, and different mutants were constructed (The Brucella strain obtained is shown in Table 1).

\section{Confocal microscope observation}

Strains were harvested through centrifugation for $1 \mathrm{~min}$ at 12,000 rpm. The sediment was suspended in $4 \%$ formaldehyde for immobilizing the cell for $20 \mathrm{~min}$. Subsequently, the sediment was resuspended in PBS. Preparing agarose pads and imaging bacteria using agarose pads [40, 41]. Image the fixed bacteria using agarose pads during the imaging process, allowing to perform color fluorescence microscopy experiments. A Zeiss LSM880 laser scanning confocal microscope (Zeiss, Germany) was used to examine the red fluorescent of the transformants.

\section{Detection of fluorescence intensity}

This approach was designed to investigate the effect of changing RBS on the fluorescence intensity of Brucella. The relative fluorescence units (RFU) of bacteria were measured using a multi-functional microplate reader (Omega FLUOstar). To reduce the error caused by the concentration of the bacterial solution, the $\mathrm{OD}_{600}$ of the bacterial solution was measured. Accordingly, the relative fluorescence intensity was expressed as RFU/OD 600 for the bacterial solution. For samples, a PBS well was used as a control.

\section{Silver staining analysis of Brucella LPS}

LPS was obtained by LPS Extraction kit (iNtRON Biotechnology, South Korea) and was quantified by LPS ELISA kit (Beijing Yanbidin technology, China). Purified LPS was separated in 12.5\% SDS-PAGE gels, and the gels were silver stained by Protein Stains Q (Sangon Biotech, China).

\section{Sensitivity of Brucella to Polymyxin B}

Strains were harvested through centrifugation for $1 \mathrm{~min}$ at 12,000 rpm. The sediment was resuspended in PBS. The final concentrations of $50,100,200$, and $500 \mu \mathrm{g} / \mathrm{mL}$ Polymyxin B were added into bacteria liquid of Brucella cultured for 1 to $5 \mathrm{~h}$ at $37^{\circ} \mathrm{C}$ and $200 \mathrm{rpm}$. Then, $100 \mu \mathrm{L}$ of the diluted solution was plated on the TSA agar medium, and the plates were incubated at $37^{\circ} \mathrm{C}$ for $72 \mathrm{~h}$. Calculate of the survival rates of mutant strains under the pressure of Polymyxin B.

\section{Statistical analysis}


All results were presented as mean \pm standard deviation (SD). Statistical analysis was performed using Prism 9.0 (GraphPad Software, CA, USA). T student test and one-way analysis of variance (ANOVA) with Dunnett's correction were applied. P-values were indicated as ns $₫ 0.05,{ }^{*} P<0.05,{ }^{\star} \mathrm{P}<0.01$, ${ }^{\star \star \star P} P<0.001$, and ${ }^{\star \star \star *} \mathrm{P}<0.0001$ indicating the significant difference. All experiments were repeated at least three times.

Table 1. Strains and plasmids used in this work. The materials were stored in the laboratory. 


\begin{tabular}{ll} 
Strain name & Description \\
\hline E. coli DH5a & General-purpose host strain for subcloning \\
\hline Brucella 104M & $\begin{array}{l}\text { B. abortus vaccine strain, originally isolated from the fetus of an } \\
\text { aborted cow in the former Soviet republic }\end{array}$ \\
\hline
\end{tabular}

Source

Lab preservation

Laboratory preservation

Brucella 16M B. melitensis strain, is the virulent reference strain.

Laboratory preservation

E. $\mathrm{MCH} / \mathrm{B}$. $\mathrm{MCH}$

(E. coli DH5a / Brucella 104M) $\mathrm{P}_{\text {sojA }}$ RBS origin sequence

This study

(GAGGTTGTGCCTGATCAGA), mCherry protein, $\mathrm{Kan}^{\mathrm{r}}$

E. $\mathrm{MCH} 1 / \mathrm{B}$. $\mathrm{MCH} 1$

(E. coli DH5a / Brucella 104M) $\mathrm{P}_{\text {sojA, }}$ RBS predict increased

This study sequence

(AAAGGAGGTCAGA), mCherry protein, $\mathrm{Kan}^{\mathrm{r}}$

E. $\mathrm{MCHO} / \mathrm{B}$. $\mathrm{MCHO}$

(E. coli DH5a / Brucella 104M) $\mathrm{P}_{\text {soja }}$, SD sequence

This study (AAAGGAGGT), mCherry protein, $\mathrm{Kan}^{\mathrm{r}}$

E. MCH4A / B.

(E. coli DH5a / Brucella 104M) $\mathrm{P}_{\text {sojA }}$ SD sequence

This study $\mathrm{MCH} 4 \mathrm{~A}$ (AAAGGAGGT), spacer sequence (AAAA), mCherry protein, Kan ${ }^{r}$

E. MCH4G / B.

(E. coli DH5a / Brucella 104M) $\mathrm{P}_{\text {soja }}$, SD sequence This study MCH4G (AAAGGAGGT), spacer sequence (GGGG), mCherry protein, $\mathrm{Kan}^{r}$

E. MCH8A / B. $\mathrm{MCH} 8 \mathrm{~A}$

(E. coli DH5a / Brucella 104M) $\mathrm{P}_{\text {sojA }}$, SD sequence This study (AAAGGAGGT), spacer sequence (AAAAAAAA), mCherry protein, $\mathrm{Kan}^{\mathrm{r}}$

E. MCH8G / B. $\mathrm{MCH} 8 \mathrm{G}$

(E. coli DH5a / Brucella 104M) $\mathrm{P}_{\text {sojA, }}$ SD sequence (AAAGGAGGT), spacer sequence (GGGGGGGG), mCherry This study protein, $\mathrm{Kan}^{\mathrm{r}}$
E. MCH12A /
B. $\mathrm{MCH} 12 \mathrm{~A}$
(E. coli DH5a / Brucella 104M) $\mathrm{P}_{\text {sojA }}$ SD sequence (AAAGGAGGT), spacer sequence (AAAAAAAAAAAA), mCherry protein, $\mathrm{Kan}^{\mathrm{r}}$

E. MCH12G /

(E. coli DH5a / Brucella 104M) $\mathrm{P}_{\text {sojA }}$ SD sequence This study

B. $\mathrm{MCH} 12 \mathrm{G}$

(AAAGGAGGT), spacer sequence (GGGGGGGGGGGG), mCherry protein, $\mathrm{Kan}^{\mathrm{r}}$

B. A1

(Brucella 16M) RBS predict increased sequence

This study (AAAGGAGGTAACC), LptA gene, $\mathrm{Kan}^{r}$

B. A101

(Brucella 16M) RBS predict increased sequence This study (AAAGGAGGTAACC), LptA gene, $\mathrm{Kan}^{\mathrm{r}}$, RBS predict increased sequence(AAAGGAGGTTGAG), $L p x O$ gene, $\mathrm{Gm}^{r}$ 


\section{Plasmid name Description}

pMAZ-SK E. coli vector, mCherry protein, $\mathrm{Kan}^{\mathrm{r}}$

pTREX- mCherry protein

mCherry

$\mathrm{pMCH} \quad \mathrm{pBM} 16 \mathrm{~A}$ T vector, $\mathrm{P}_{\text {sojA }}$, RBS origin sequence

(GAGGTTGTGCCTGATCAGA), mCherry protein, $\mathrm{Kan}^{\mathrm{r}}$

$\mathrm{pMCH} 1$

pBM16A T vector, $\mathrm{P}_{\text {sojA, }}$ RBS predict increased sequence (AAAGGAGGTCAGA), mCherry protein, $\mathrm{Kan}^{\mathrm{r}}$

$\mathrm{pMCHO}$

pBM16A T vector, $\mathrm{P}_{\text {sojA }}$, SD sequence AAAGGAGGT, mCherry protein, $\operatorname{Kan}^{r}$

$\mathrm{pMCH} 4 \mathrm{~A}$

pBM16A T vector, $\mathrm{P}_{\text {sojA, }}$ SD sequence AAAGGAGGT, spacer sequence (AAAA), mCherry protein, $\mathrm{Kan}^{\mathrm{r}}$

$\mathrm{pMCH} 4 \mathrm{G}$

pBM16A T vector, $P_{\text {sojA, }}$ SD sequence AAAGGAGGT, spacer sequence (GGGG), mCherry protein, $\mathrm{Kan}^{\mathrm{r}}$

$\mathrm{pMCH8A}$

pBM16A T vector, $P_{\text {sojA, }}$ SD sequence AAAGGAGGT, spacer sequence (AAAAAAAA), mCherry protein, $\mathrm{Kan}^{\mathrm{r}}$

pMCH8G

pBM16A T vector, $P_{\text {sojA }}$, SD sequence AAAGGAGGT, spacer sequence (GGGGGGGG), mCherry protein, $\mathrm{Kan}^{\mathrm{r}}$

pMCH12A

pBM16A T vector, $\mathrm{P}_{\text {sojA }}$, SD sequence AAAGGAGGT, spacer sequence (AAAAAAAAAAAA), mCherry protein, $\operatorname{Kan}^{r}$

$\mathrm{pMCH} 12 \mathrm{G}$

pBM16A T vector, $\mathrm{P}_{\text {sojA }}$, SD sequence AAAGGAGGT, spacer sequence (GGGGGGGGGGGG), mCherry protein, $\mathrm{Kan}^{\mathrm{r}}$

pA1

pA101
pBM16A T vector, RBS predict increased sequence (AAAGGAGGTAACC), LptA gene, $\mathrm{Kan}^{\mathrm{r}}$

pBM16A T vector, RBS predict increased sequence (AAAGGAGGTAACC), LptA gene, $\mathrm{Kan}^{\mathrm{r}}$, RBS predict increased sequence (AAAGGAGGTTGAG), LpxO gene, $\mathrm{Gm}^{r}$
Source

Laboratory preservation[37]

Laboratory preservation[38, 39]

This study

This study

This study

This study

This study

This study

This study

This study

This study

This study

This study

$A m p^{r}$, Resistance to ampicillin; Kan ${ }^{r}$, Resistance to kanamycin; $\mathrm{Gm}^{r}$, Resistance to gentamicin

\section{Abbreviations}

LPS: Lipopolysaccharide; PAMP: Pathogen associated molecular pattern; mRNA: Messenger RNA; rRNA: Ribosomal RNA; RBS: Ribosomal binding site; SD sequence: Shine-Dalgarno sequence; E. coli: 
Escherichia coli; B. subtilis: Bacillus subtilis; UTR: Untranslated region; LB: Luria bertani; $B$. abortus: Brucella abortus, B. melitensis: Brucella melitensis; TSA: Trypticase soy agar; TSB: Trypticase soy broth; $\mathrm{Kan}^{\mathrm{r}}$ : Resistance to kanamycin; $\mathrm{Gm}^{\mathrm{r}}$ : Resistance to gentamicin; Ampr: Resistance to ampicillin; BSC: Biological safety cabinet; DNA: Deoxyribonucleic acid; PCR: Polymerase chain reaction; RFU: relative fluorescence units; OD: Optical density; SDS-PAGE: Sodium dodecyl sulfate-polyacrylamide gelelectrophoresis; SD: standard deviation.

\section{Declarations}

\section{Acknowledgements}

Not applicable.

\section{Authors' contributions}

Conceptualized the idea: LZ and SL. Experiments designed: LZ, SL, PW, SL. Experiments conducted: SL. Data analyzed and results discussed: SL, PW, HX. Manuscript written: SL, PW, YD. Edited, reviewed, and approved: LZ and YD. All the authors helped in reviewing the whole manuscript. All authors read and approved the final manuscript. SL and PW contributed equally to this article.

\section{Funding}

This work was supported by Jilin Scientific and Technological Development Program (20180101264JC).

\section{Availability of data and materials}

All data generated or analyzed during this study are included in this published article.

\section{Ethics approval and consent to participate}

Not applicable.

\section{Consent for publication}

Not applicable.

\section{Competing interests}

The authors declare that they have no competing interests.

\section{References}

1. Martínez-Gómez E, Ståhle J, Gil-Ramírez Y, Zúñiga-Ripa A, Zaccheus M, Moriyón I, Iriarte M, Widmalm G, Conde-Álvarez R: Genomic Insertion of a Heterologous Acetyltransferase Generates a New 
Lipopolysaccharide Antigenic Structure in Brucella abortus and Brucella melitensis. Front Microbio/ 2018, 9:1092.

2. Feng $Y$, Peng $X$, Jiang $H$, Peng $Y$, Zhu L, Ding J: Rough brucella strain RM57 is attenuated and confers protection against Brucella melitensis. Microb Pathog 2017, 107:270-275.

3. Suárez-Esquivel M, Chaves-Olarte E, Moreno E, Guzmán-Verri C: Brucella Genomics: Macro and Micro Evolution. Int J Mol Sci 2020, 21.

4. Aragón-Aranda B, de Miguel MJ, Lázaro-Antón L, Salvador-Bescós M, Zúñiga-Ripa A, Moriyón I, Iriarte M, Muñoz PM, Conde-Álvarez R: Development of attenuated live vaccine candidates against swine brucellosis in a non-zoonotic B. suis biovar 2 background. Vet Res 2020, 51:92.

5. Barbier T, Zúñiga-Ripa A, Moussa S, Plovier H, Sternon JF, Lázaro-Antón L, Conde-Álvarez R, De Bolle $\mathrm{X}$, Iriarte M, Moriyón I, Letesson JJ: Brucella central carbon metabolism: an update. Crit Rev Microbiol 2018, 44:182-211.

6. Minor PD: Live attenuated vaccines: Historical successes and current challenges. Virology 2015, 479480:379-392.

7. Zhao Y, Arce-Gorvel V, Conde-Álvarez R, Moriyon I, Gorvel JP: Vaccine development targeting lipopolysaccharide structure modification. Microbes Infect 2018, 20:455-460.

8. Lapaque N, Moriyon I, Moreno E, Gorvel JP: Brucella lipopolysaccharide acts as a virulence factor. Curr Opin Microbiol 2005, 8:60-66.

9. Tupik JD, Coutermarsh-Ott SL, Benton AH, King KA, Kiryluk HD, Caswell CC, Allen IC: ASC-Mediated Inflammation and Pyroptosis Attenuates Brucella abortus Pathogenesis Following the Recognition of gDNA. Pathogens 2020, 9.

10. Conde-Álvarez R, Palacios-Chaves L, Gil-Ramírez Y, Salvador-Bescós M, Bárcena-Varela M, AragónAranda B, Martínez-Gómez E, Zúñiga-Ripa A, de Miguel MJ, Bartholomew TL, et al: Identification of IptA, IpxE, and IpxO, Three Genes Involved in the Remodeling of Brucella Cell Envelope. Front Microbio/ 2017, 8:2657.

11. Petrov A, Chen J, O'Leary S, Tsai A, Puglisi JD: Single-molecule analysis of translational dynamics. Cold Spring Harb Perspect Biol 2012, 4:a011551.

12. Brenner S, Jacob F, Meselson M: An unstable intermediate carrying information from genes to ribosomes for protein synthesis. Nature 1961, 190:576-581.

13. Leppek K, Fujii K, Quade N, Susanto TT, Boehringer D, Lenarčič T, Xue S, Genuth NR, Ban N, Barna M: Gene- and Species-Specific Hox mRNA Translation by Ribosome Expansion Segments. Mol Cell 2020, 80:980-995.e913. 
14. Wan X, Pinto F, Yu L, Wang B: Synthetic protein-binding DNA sponge as a tool to tune gene expression and mitigate protein toxicity. Nat Commun 2020, 11:5961.

15. Lin J, Zhou D, Steitz TA, Polikanov YS, Gagnon MG: Ribosome-Targeting Antibiotics: Modes of Action, Mechanisms of Resistance, and Implications for Drug Design. Annu Rev Biochem 2018, 87:451478.

16. Romilly C, Deindl S, Wagner EGH: The ribosomal protein S1-dependent standby site in tisB mRNA consists of a single-stranded region and a 5' structure element. Proc Natl Acad Sci U S A 2019, 116:15901-15906.

17. Laursen BS, Sørensen HP, Mortensen KK, Sperling-Petersen HU: Initiation of protein synthesis in bacteria. Microbiol Mol Biol Rev 2005, 69:101-123.

18. Brenneis M, Soppa J: Regulation of translation in haloarchaea: 5'- and 3'-UTRs are essential and have to functionally interact in vivo. PLoS One 2009, 4:e4484.

19. Volkenborn K, Kuschmierz L, Benz N, Lenz P, Knapp A, Jaeger KE: The length of ribosomal binding site spacer sequence controls the production yield for intracellular and secreted proteins by Bacillus subtilis. Microb Cell Fact 2020, 19:154.

20. Jha V, Roy B, Jahagirdar D, McNutt ZA, Shatoff EA, Boleratz BL, Watkins DE, Bundschuh R, Basu K, Ortega J, Fredrick K: Structural basis of sequestration of the anti-Shine-Dalgarno sequence in the Bacteroidetes ribosome. Nucleic Acids Res 2021, 49:547-567.

21. Salis HM, Mirsky EA, Voigt CA: Automated design of synthetic ribosome binding sites to control protein expression. Nat Biotechnol 2009, 27:946-950.

22. Scharff LB, Childs L, Walther D, Bock R: Local absence of secondary structure permits translation of mRNAs that lack ribosome-binding sites. PLoS Genet 2011, 7:e1002155.

23. Bentele K, Saffert P, Rauscher R, Ignatova Z, Blüthgen N: Efficient translation initiation dictates codon usage at gene start. Mol Syst Biol 2013, 9:675.

24. de Smit MH, van Duin J: Control of prokaryotic translational initiation by mRNA secondary structure. Prog Nucleic Acid Res Mol Biol 1990, 38:1-35.

25. Goltermann $L$, Borch Jensen $M$, Bentin $T$ : Tuning protein expression using synonymous codon libraries targeted to the 5' mRNA coding region. Protein Eng Des Sel 2011, 24:123-129.

26. Kudla G, Murray AW, Tollervey D, Plotkin JB: Coding-sequence determinants of gene expression in Escherichia coli. Science 2009, 324:255-258. 
27. Na D, Lee S, Lee D: Mathematical modeling of translation initiation for the estimation of its efficiency to computationally design mRNA sequences with desired expression levels in prokaryotes. $B M C$ Syst Biol 2010, 4:71.

28. Hartz D, McPheeters DS, Gold L: Influence of mRNA determinants on translation initiation in Escherichia coli. J Mol Biol 1991, 218:83-97.

29. Osterman IA, Evfratov SA, Sergiev PV, Dontsova OA: Comparison of mRNA features affecting translation initiation and reinitiation. Nucleic Acids Res 2013, 41:474-486.

30. Vellanoweth RL, Rabinowitz JC: The influence of ribosome-binding-site elements on translational efficiency in Bacillus subtilis and Escherichia coli in vivo. Mol Microbiol 1992, 6:1105-1114.

31. Vimberg $\mathrm{V}$, Tats $\mathrm{A}$, Remm $\mathrm{M}$, Tenson $\mathrm{T}$ : Translation initiation region sequence preferences in Escherichia coli. BMC Mol Biol 2007, 8:100.

32. Kohler S, Foulongne V, Ouahrani-Bettache S, Bourg G, Teyssier J, Ramuz M, Liautard JP: The analysis of the intramacrophagic virulome of Brucella suis deciphers the environment encountered by the pathogen inside the macrophage host cell. Proc Natl Acad Sci U S A 2002, 99:15711-15716.

33. Köhler S, Ouahrani-Bettache S, Layssac M, Teyssier J, Liautard JP: Constitutive and inducible expression of green fluorescent protein in Brucella suis. Infect Immun 1999, 67:6695-6697.

34. Shaner NC, Campbell RE, Steinbach PA, Giepmans BN, Palmer AE, Tsien RY: Improved monomeric red, orange and yellow fluorescent proteins derived from Discosoma sp. red fluorescent protein. Nat Biotechnol 2004, 22:1567-1572.

35. Liebeton $\mathrm{K}$, Lengefeld J, Eck J: The nucleotide composition of the spacer sequence influences the expression yield of heterologously expressed genes in Bacillus subtilis. J Biotechno/2014, 191:214-220.

36. Copin R, Vitry MA, Hanot Mambres D, Machelart A, De Trez C, Vanderwinden JM, Magez S, Akira S, Ryffel B, Carlier Y, et al: In situ microscopy analysis reveals local innate immune response developed around Brucella infected cells in resistant and susceptible mice. PLoS Pathog 2012, 8:e1002575.

37. Ronda C, Pedersen LE, Sommer MO, Nielsen AT: CRMAGE: CRISPR Optimized MAGE Recombineering. Sci Rep 2016, 6:19452.

38. O'Halloran DM: A Guide to Using STITCHER for Overlapping Assembly PCR Applications. Methods Mol Biol 2017, 1472:3-11.

39. Lu Y, Xiao S, Yuan M, Gao Y, Sun J, Xue C: Using overlap-extension PCR technique to fusing genes for constructing recombinant plasmids. J Basic Microbiol 2018, 58:273-276. 
40. Skinner SO, Sepúlveda LA, Xu H, Golding I: Measuring mRNA copy number in individual Escherichia coli cells using single-molecule fluorescent in situ hybridization. Nat Protoc 2013, 8:1100-1113.

41. Arbel-Goren R, Shapira Y, Stavans J: Method for Labeling Transcripts in Individual Escherichia coli Cells for Single-molecule Fluorescence In Situ Hybridization Experiments. J Vis Exp 2017.

\section{Figures}




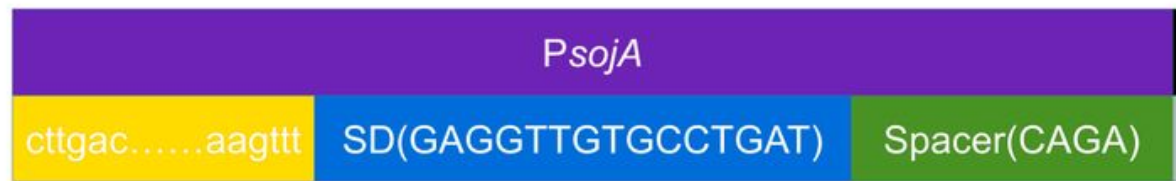

\section{target gene}

$3^{\prime}$

SecE(ATG

$\downarrow$ replacement $\downarrow$ insertion

mCherry(ATG......) $K a n^{r}$

b

\section{5 ,}

\section{SD}

spacer

mCherry-Kanr

3' plasmid

...acgcgt...TTT GAGGTTGTGCCTGAT

..CAGA ATG...tggcca....tgcag...

$\mathrm{pMCH}$

....acgcgt...TTT .AAAGGAGGT ..CAGA

ATG...tggcca...ctgcag... pMCH1

...acgcgt...TTT AAAGGAGGT

ATG...tggcca...ctgcag...

pMCHO

...acgcgt...TTT .AAAGGAGGT .AAAA ATG...tggcca....ctgcag... $\mathrm{pMCH} 4 \mathrm{~A}$

...acgcgt...TTT AAAGGAGGT ..GGGG ATG...tggcca...ctgcag... pMCH4G

....acgcgt...TTT AAAGGAGGT AAAAAAAA ATG...tggcca...ctgcag... PMCH8A

...acgcgt...TTT AAAGGAGGT GGGGGGGG

ATG...tggcca...ctgcag... .AAAGGAGGT ..AAAAAAAAAAAA ATG...tggcca....tgcag... pMCH8G

...acgcgt...TTT AAAGGAGGT GGGGGGGGGGGG

...acgcgt...TTT

................

AfIII

ATG...tggcca...ctgcag...

pMCH12A

pMCH12G

\section{c}

5 ,

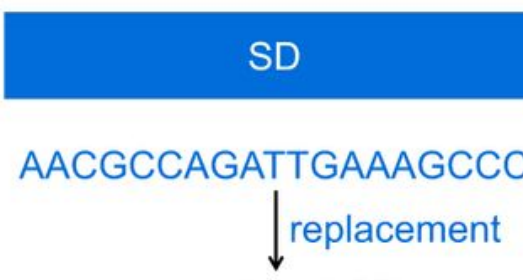

...aagatc

AAAGGAGGT

AACC
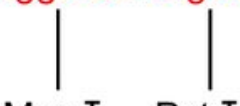

Msc I Pst I

...aagatc AACGCCAGATTGAAAGCCC AACC

d 5 ,

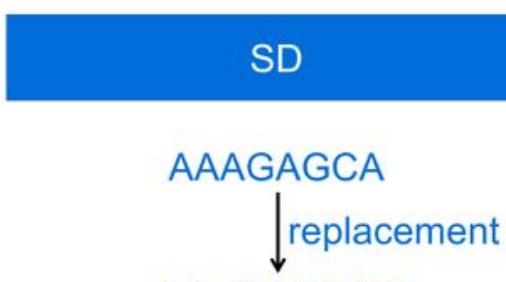

...gttttg

AAAGGAGGT

\section{spacer}

TGAG

...gttttg

$\downarrow$ replacement

c...
TGAG

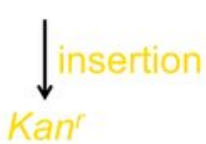

pA1

3' plasmid

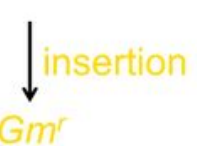

p01

\section{Figure 1}

Nucleotide composition of RBS of plasmids. (a)The expression of target gene (black) was controlled by a strong Brucella spp. promoter called PsojA (purple). SD sequence (blue), spacer (green) and start of SecE (grey) were highlighted. The mCherry gene (red) was integrated into the genome of Brucella 104M to replace the SecE gene, and the new strain was named $\mathrm{B}$. $\mathrm{MCH}$; (b) Nucleotide composition of RBS of plasmids pMCH, pMCH1, pMCH0, pMCH4A, pMCH4G, pMCH8A, pMCH8G, pMCH12A, pMCH12G. SD 
sequence (blue), spacer (green) and start of target gene (red) were highlighted. The cleavage sites of

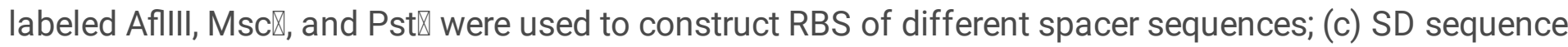
(blue), spacer (green) and start of LptA (yellow) were highlighted. The SD sequence (blue) aacgccagattgaaagccc was replaced by AAAGGAGGT, and the new plasmid was named pA1; (d) SD sequence (blue), spacer (green) and start of LpxO (yellow) were highlighted. The SD sequence (blue) aaagagca was replaced by AAAGGAGGT, and the new plasmid was named p01.

a

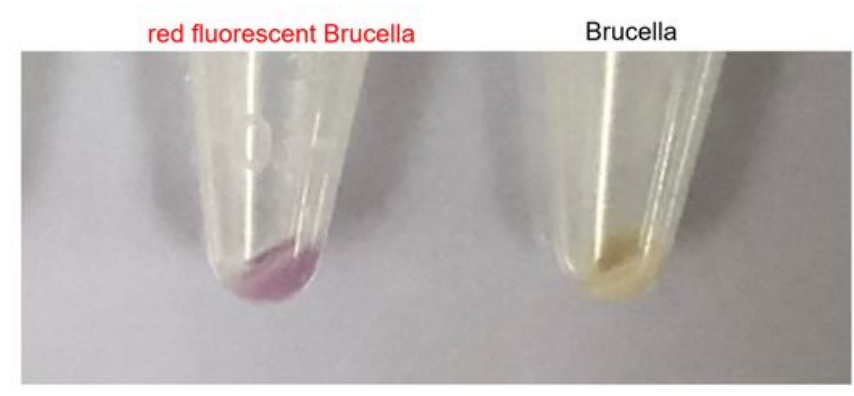

b

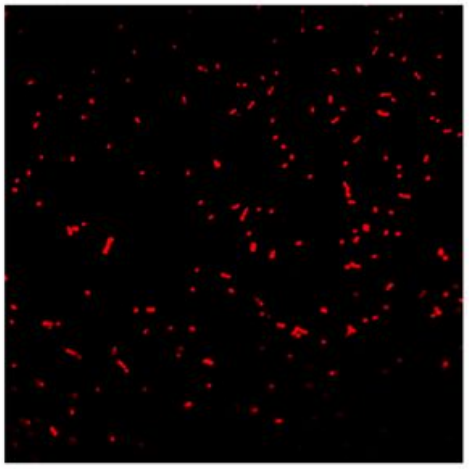

C

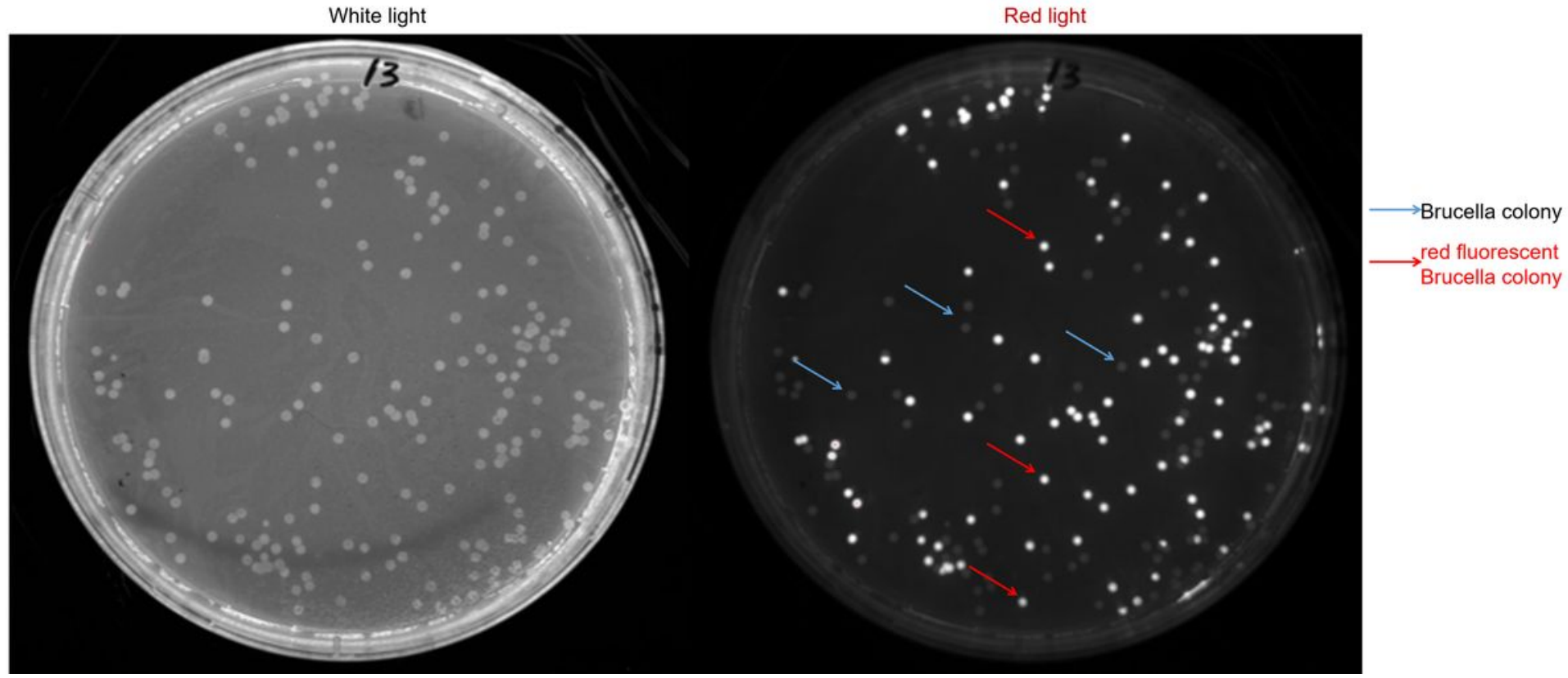

Figure 2

Images of strains with red fluorescent protein. (a) naked-eye; (b) fluorescence microscope; (c) multifunction imager. 
a
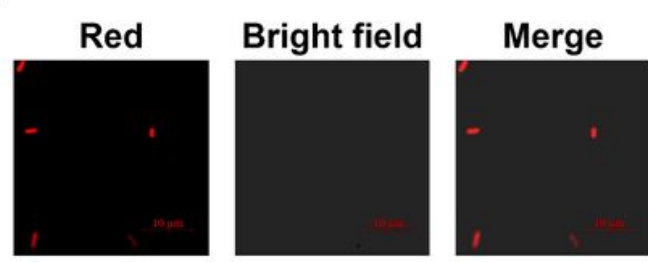

E. $\mathrm{MCH}$
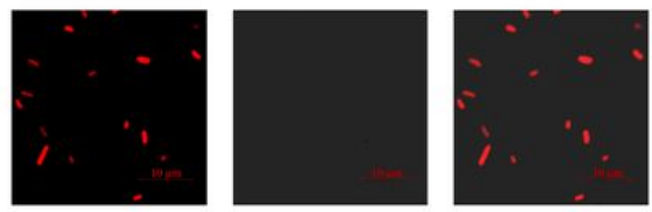

E. $\mathrm{MCH} 1$

C

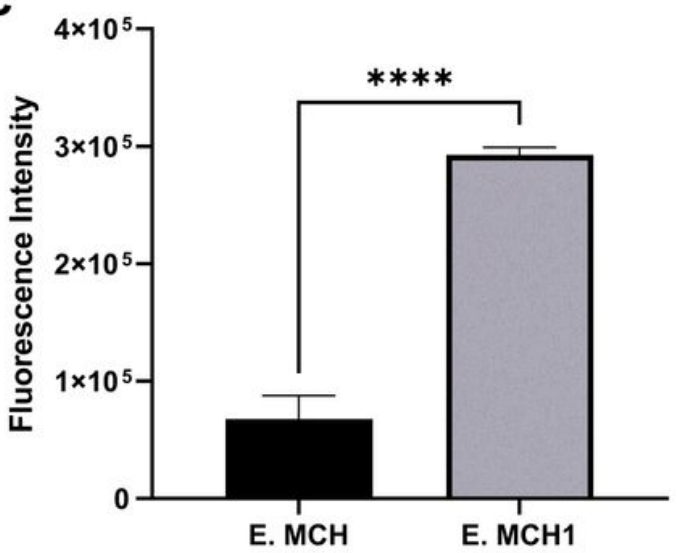

b

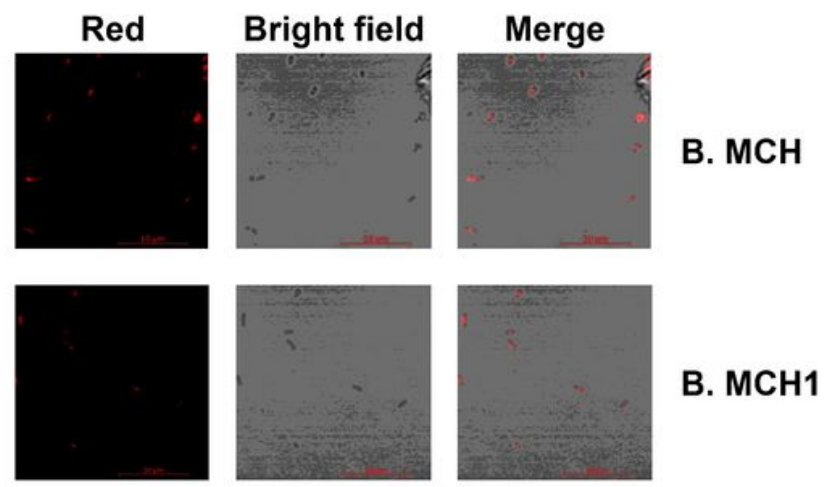

d

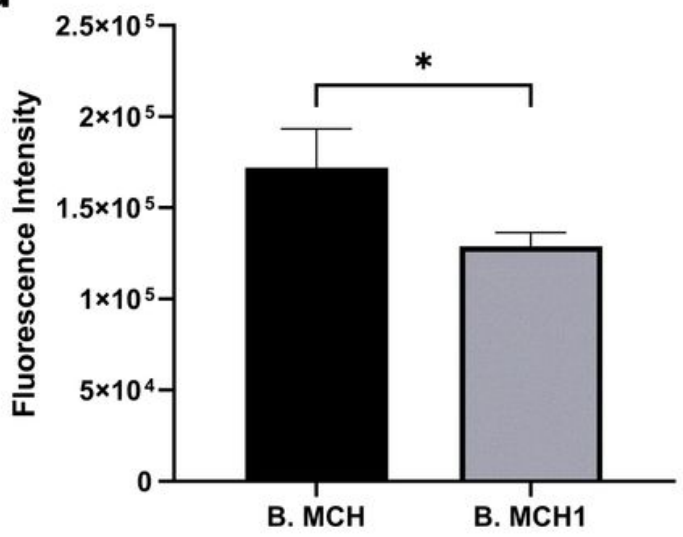

Figure 3

Effect of SD sequences on gene expression in E. coli and Brucella. (a) The difference of fluorescence intensity between $\mathrm{E}$. $\mathrm{MCH}$ and $\mathrm{E} . \mathrm{MCH} 1$. ${ }^{\star \star \star *}, \mathrm{P}<0.0001$; (b) The difference of fluorescence intensity between $\mathrm{B}$. MCH and $\mathrm{B}$. MCH1. *, $\mathrm{P}<0.05$; (c) Images of confocal microscope showed red fluorescence of E. $\mathrm{MCH}$ and $\mathrm{E}$. MCH1; (d) Images of confocal microscope showed red fluorescence of $\mathrm{B}$. MCH and $\mathrm{B}$. $\mathrm{MCH} 1$. 
a
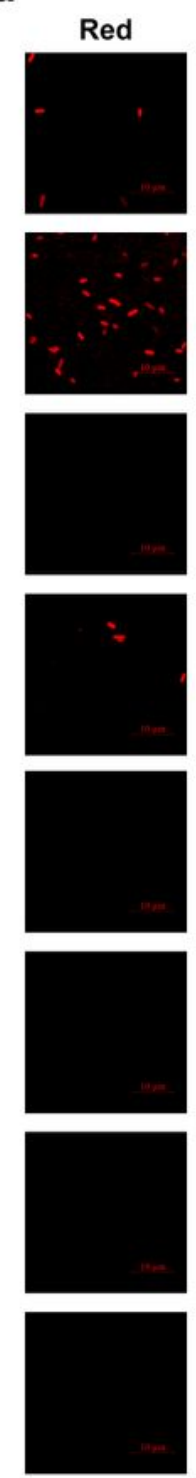

C
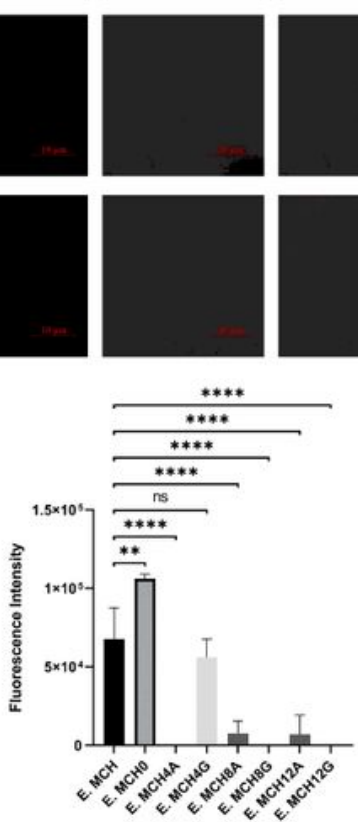

b

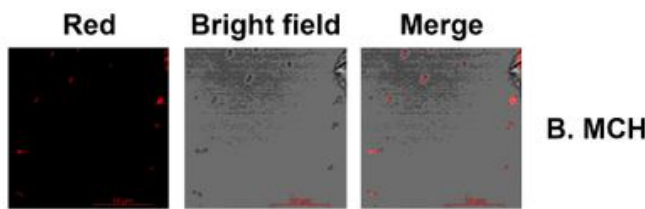

E. MCHO
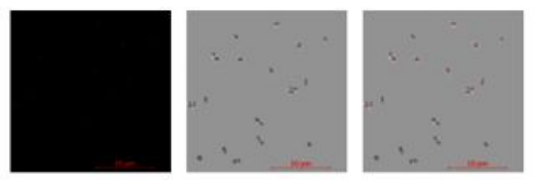

B MCHO
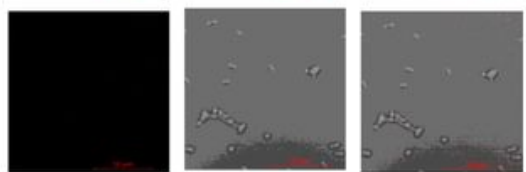

B. $\mathrm{MCH} 4 \mathrm{~A}$
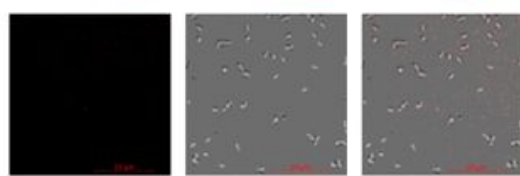

B. $\mathrm{MCH} 4 \mathrm{G}$
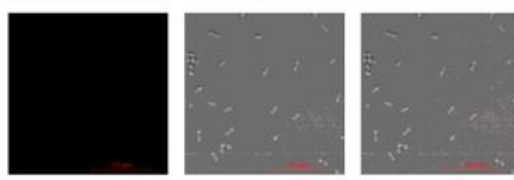

B. $\mathrm{MCH} 8 \mathrm{~A}$
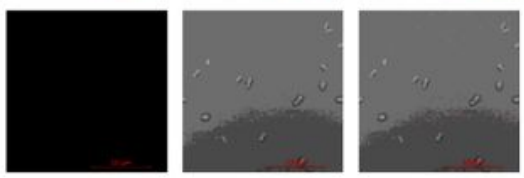

B. $\mathrm{MCH} 8 \mathrm{G}$

E. MCH8G

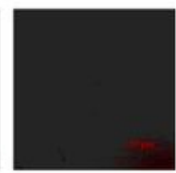

E. MCH12A
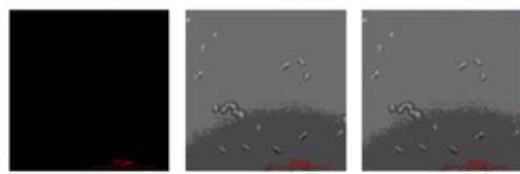

B. $\mathrm{MCH} 12 \mathrm{~A}$
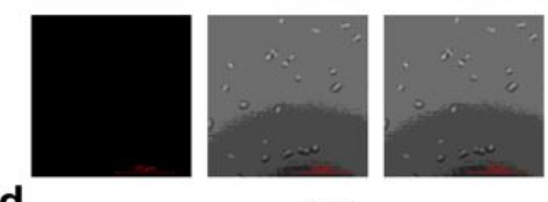

B. $\mathrm{MCH} 12 \mathrm{G}$

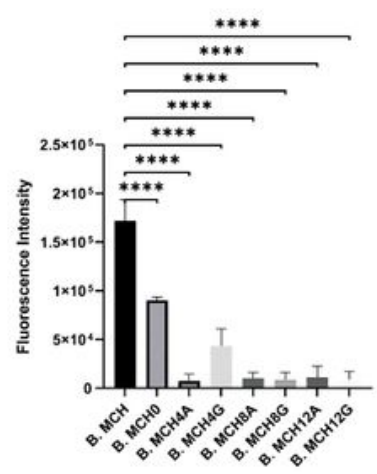

Figure 4

Effect of spacer sequences on gene expression in E. coli and Brucella. (a) The difference of fluorescence intensity between E. MCH and E. MCH0, E. MCH4A, E. MCH4G, E. MCH8A, E. MCH8G, E. MCH12A, E.

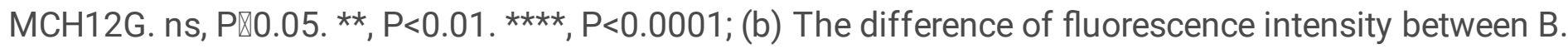

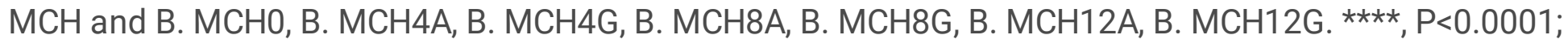
(c) Images of confocal microscope showed red fluorescence of $\mathrm{E} . \mathrm{MCH}$ and $\mathrm{E}$. MCH0, E. MCH4A, E. 
MCH4G, E. MCH8A, E. MCH8G, E. MCH12A, E. MCH12G; (d) Images of confocal microscope showed red fluorescence of B. MCH and B. MCH0, B. MCH4A, B. MCH4G, B. MCH8A, B. MCH8G, B. MCH12A, B. MCH12G.

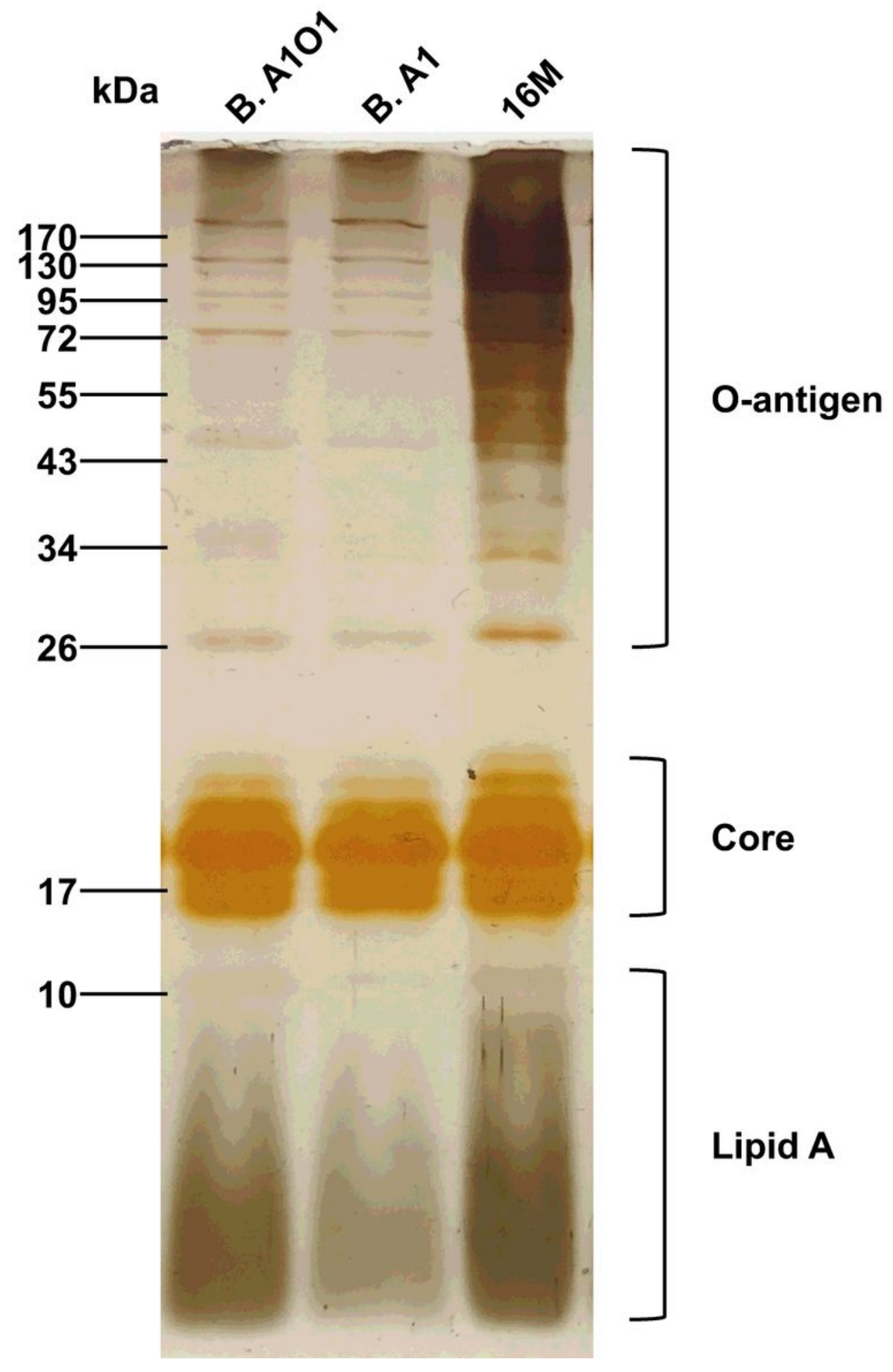

Figure 5

The picture of SDS-PAGE gel of silver staining. The LPS, after extracted from the Brucella mutant strains, was visualized by silver staining. LPS consists of $\mathrm{O}$-antigen, Core, and Lipid A. The breadth and color of 
O-antigen, Core and Lipid A of the LPS of B. A1 and B. A101 were weaker than $16 \mathrm{M}$.

a

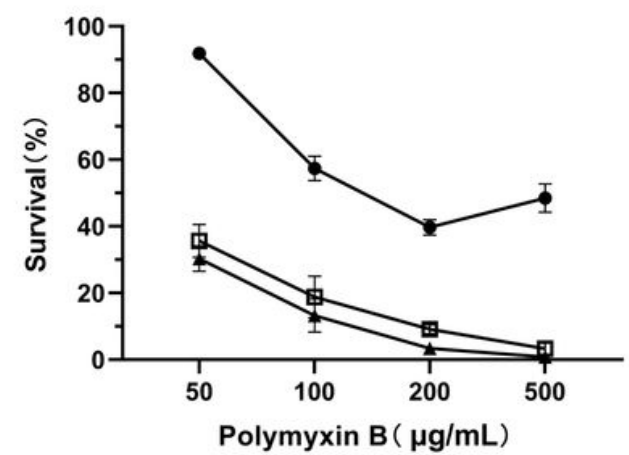

C

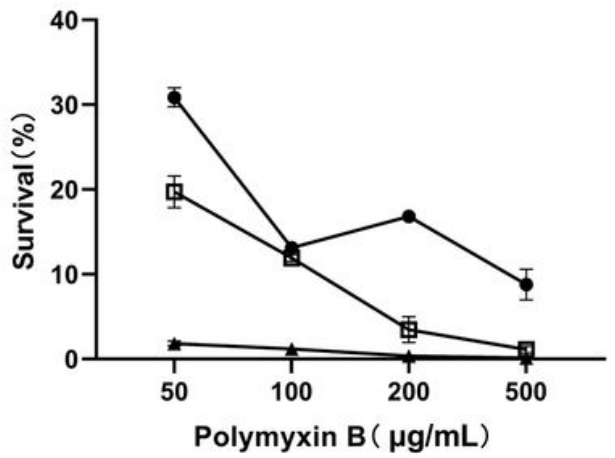

e

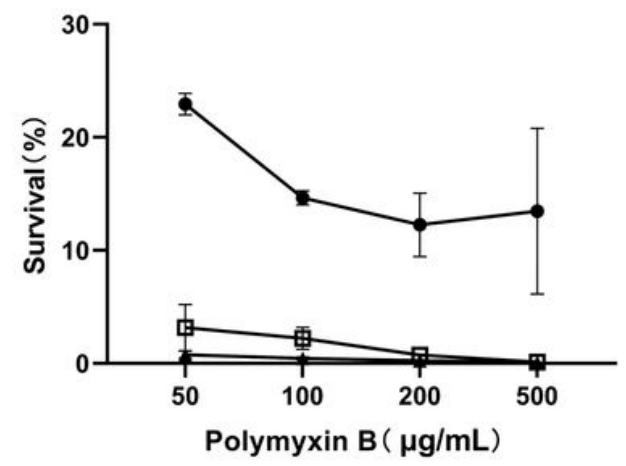

b

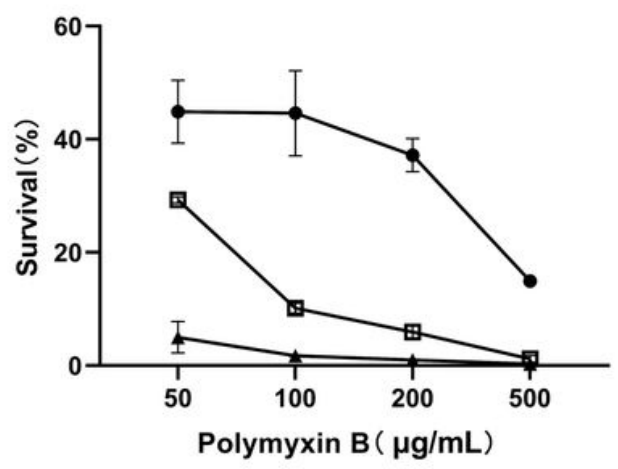

$\rightarrow 16 \mathrm{M}$

廿 B. A1

- B. A101

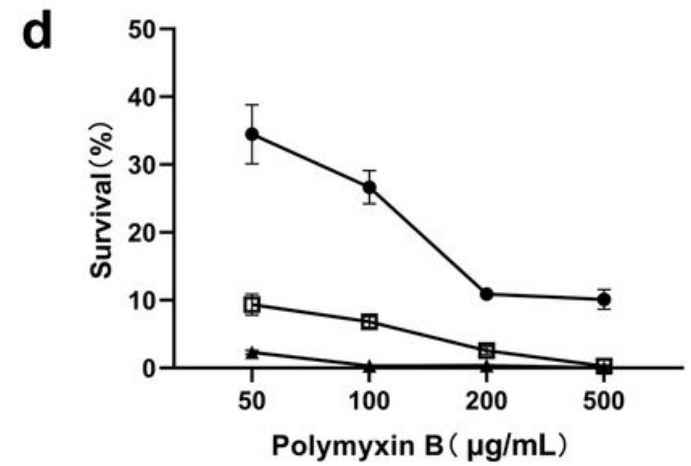

$\rightarrow 16 \mathrm{M}$

廿 B. A1

- B. A101 $\rightarrow 16 \mathrm{M}$

廿 B. A1

- B. A101

Figure 6

Survival curves of Brucella under the treatment of Polymyxin B at concentrations of 50, 100, 200, and 500 $\mu \mathrm{g} / \mathrm{mL}$ for $1 \mathrm{~h}(\mathrm{a}), 2 \mathrm{~h}(\mathrm{~b}), 3 \mathrm{~h}(\mathrm{c}), 4 \mathrm{~h}(\mathrm{~d})$ and $5 \mathrm{~h}(\mathrm{e})$.

\section{Supplementary Files}

This is a list of supplementary files associated with this preprint. Click to download.

- Tables1.docx 